\title{
NPH Log: Validation of a New Assessment Tool Leading to Earlier Diagnosis of Normal Pressure Hydrocephalus
}

Ignacio Jusué-Torres ${ }^{1}$, Jennifer $\mathrm{Lu}^{2}$, Jamie Robison ${ }^{2}$, Jamie B. Hoffberger ${ }^{2}$, Alicia Hulbert ${ }^{3}$, Abanti Sanyal ${ }^{4}$, Jan Wemmer ${ }^{2}$, Benjamin D. Elder ${ }^{2}$, Daniele Rigamonti ${ }^{2}$

1. Department of Neurosurgery, Loyola University Chicago, Stritch School of Medicine, Maywood, Illinois 2. Department of Neurosurgery, The Johns Hopkins University School of Medicine 3. Department of Oncology, The Johns Hopkins University School of Medicine 4. Department of Biostatistics, Johns Hopkins Bloomberg School of Public Health

$\square$ Corresponding author: Ignacio Jusué-Torres, ignaciojusue@gmail.com Disclosures can be found in Additional Information at the end of the article

\section{Abstract}

Introduction:

Early treatment of normal pressure hydrocephalus (NPH) yields better postoperative outcomes. Our current tests often fail to detect significant changes at early stages. We developed a new scoring system (LP log score) to determine if this tool is more sensitive in detecting clinical differences than current tests.

Material and Methods:

Sixty-two consecutive new patients with suspected idiopathic NPH were studied. Secondary, previously treated and obstructive cases were not included. We collected age, pre- and postlumbar puncture (LP) Tinetti, Timed Up and Go (TUG) Test, European NPH scale, and LP log scores. The LP log score is recorded at baseline and for seven consecutive days after removing 40 cc of cerebrospinal fluid (CSF) via LP. We studied the diagnostic accuracy of the tests for surgical indication.

Results:

The post-LP log showed improvement in 90\% of people with good baseline gait tests and in 93\% of people who did not show any pre-LP and post-LP change in gait tests. Sensitivity, specificity, and accuracy to detect intention to treat when positive post-LP improvements were $4 \%, 100 \%$, and $24 \%$, respectively, for TUG, $21 \%$, $86 \%$, and $34 \%$, respectively, for the Tinetti Mobility Test, 66\%, 29\%, and 58\%, respectively, for Medical College of Virginia (MCV) grade, and $98 \%, 33 \%$, and $85 \%$, respectively, for LP log score. Pre-LP and post-LP TUG improvement and pre-LP and post-LP Tinetti improvement were not associated with a surgical indication $(\mathrm{p}>$ 0.05). LP log improvement was associated with surgical indication odds ratio (OR): $24.595 \% \mathrm{CI}$ $(2.4-248.12)(\mathrm{p}=0.007)$.

Conclusions:

LP log showed better sensitivity, diagnostic accuracy, and association with surgical indication than the current diagnostic approach. An LP log may be useful detecting NPH patients at earlier stages and, therefore, yield better surgical outcomes. 
Categories: Neurology, Neurosurgery

Keywords: normal pressure hydrocephalus, early diagnosis, sensitivity, specificity, diagnostic accuracy

\section{Introduction}

In 1965, Solomon Hakim described normal pressure hydrocephalus (NPH) as "a mild impairment of memory, slowness, and paucity of thought and action, unsteadiness of gait, and unwitting urination" [1-2]. Hakim's triad, considered pathognomic of NPH, refers to the contemporaneous presence of gait, cognition, and urinary difficulties in the context of ventriculomegaly [1-2]. Many neurologists and neurosurgeons, therefore, consider the diagnosis of iNPH only in the presence of the three symptoms.

In reality, many patients that benefited from surgery for hydrocephalus do not present with the full "triad." The complete syndrome may correspond to a late phase of this condition [3-6], as asymptomatic ventriculomegaly observed on magnetic resonance imaging (MRI) may precede the development of the characteristic symptomatology by several years [7]. Furthermore, patients treated for iNPH early after the onset of their symptoms have better postoperative outcomes $[6,8]$. The major difficulty is that our current tests often fail to detect significant changes at early stages. The European iNPH Multicentre Study showed that while the resistance to CSF outflow and the CSF tap test have high positive predictive values (PPV > 90\%), they cannot exclude patients for surgery, given their very low negative predictive values (NPV < 20\%) [9]. Therefore, more sensitive tests are clearly needed to detect iNPH at earlier stages.

To achieve more sensitive testing, an iNPH assessment (LP log) was developed to quantitatively measure this subjective performance deterioration. The objective of this study was to validate this tool and demonstrate as a "proof of concept" that is more sensitive in detecting clinical differences than the currently available testing modalities.

\section{Materials And Methods}

Following approval from the Institutional Review Board (NA_00044584), a retrospective review of prospectively collected data was performed. Patient and caregiver consents were obtained before enrollment to the study. The records of all new patients referred to the Hydrocephalus Clinic at a single-institution for evaluation of suspected iNPH from 2013 to 2015 were recorded.

Inclusion criteria comprised age greater than 60 years, clinical symptoms suggestive of nonpreviously treated idiopathic hydrocephalus, Evans Index greater than 0.3, and normal morphology of the third ventricle. Patients with a known cause of hydrocephalus, such as trauma, tumor, infection, or bleeding, were excluded. Other exclusion criteria were patients with obstructive hydrocephalus or evidence of bowing of the third ventricular floor and/or the anterior wall of the third ventricle [10] or if patients were previously treated for iNPH with shunting or endoscopic third ventriculostomy.

Demographic factors and clinical information were prospectively collected. Demographic factors included: age, gender, race, and body mass index (BMI). Clinical information included the presence of gait/balance impairment, urinary urgency/incontinence, memory/attention deficits, headaches/dizziness, Hakim's Triad, and duration of symptoms at presentation. Forty cc of CSF were removed via lumbar puncture (LP) for evaluation of suspected hydrocephalus in all patients. Pre-LP and post-LP functional status were assessed using the Timed Up and Go (TUG) test, Tinetti score, Medical College of Virginia (MCV) gait grade (1 to 6), Mini-Mental State Examination (MMSE), and European iNPH Multicentre Study [5]. TUG and MCV lower values suggest better gait performance. For Tinetti score, MMSE, and European Hydrocephalus 


\section{Cureus}

Scale, higher values reflect better status.

In the clinic, before performing the LP, the patients and caregivers were routinely asked to measure the patient's baseline performance status using questions in the new LP log assessment form, as shown in Tables 1-2.

\section{Strongly}

Agree
Strongly

Disagree

1. I feel balanced.

2. I feel confident walking inside and outside.

3. I am able to stand up and sit down with ease.

4. I am able to walk up and down stairs and or hills with ease.

5. I have energy each day to complete my daily tasks.

6. I am easily able to make plans, problem solve, and move from one task to the next.

7. I am easily able to pay close and continuous attention to tasks.

8. I have the motivation to perform daily chores, errands, and call or see my family and friends.

9. I enjoy listening to music.

10. I have issues with my urinary urgency.

11. In the past $\mathbf{3}$ months, I feel that I can process questions/commands/requests that are made to me, and react appropriately to them without delay or needing of repetition

TABLE 1: Patient's Baseline - Normal Pressure Hydrocephalus Log 


\section{Cureus}

\section{\begin{tabular}{l|l|l|l|l|}
$\begin{array}{l}\text { Strongly } \\
\text { Agree }\end{array}$ & Agree & Unsure & Disagree & $\begin{array}{l}\text { Strongly } \\
\text { Disagree }\end{array}$ \\
\hline
\end{tabular}}

1. The patient is balanced.

2. The patient is confident walking inside and outside.

3. The patient is able to stand up and sit down with ease.

4. The patient is able to walk up and down stairs and or hills with ease.

5. The patient has energy each day to complete their daily tasks.

6. The patient is easily able to problem solve and move from one task to the next.

7. The patient is easily able to pay close and continuous attention to tasks.

8. The patient has the motivation to do daily chores, errands, call or see their family and friends.

9. The patient enjoys listening to music.

10. The patient has issues with their urinary urgency.

11. In the past 3 months, the patient seems to be able to process questions/commands/requests made to them, and can react appropriately with no delay or needing of repetition

TABLE 2: Caregiver's Baseline - Normal Pressure Hydrocephalus Log

Following the high-volume LP, the patients and caregivers measured the patient's change in performance using the same questions used for the baseline assessment for seven consecutive days, as shown in Tables 3-4. The patients and caregivers were asked to state if they "strongly agreed, agreed, were unsure, disagreed, or strongly disagreed" with 11 statements describing patient's function regarding activities of daily living. 


\section{Cureus}

Day Day Day Day Day Day Day

\begin{tabular}{ll|l|l|l|l|l}
1 & 2 & 3 & 4 & 5 & 6 & 7
\end{tabular}

1. I am more balanced.

2. I am more confident walking inside and outside.

3. I am better able to stand up and sit down.

4. I am better able to walk up and down stairs and hills.

5. I have more energy to complete my daily tasks.

6. I am better able to make plans, problem solve, and move from one task to the next.

7. I am better able to pay close and continuous attention to tasks.

8. I have more motivation to do daily chores and errands, call or see friends and family.

9. I enjoyed listening to music more.

10. I have fewer issues with my urinary urgency.

11. I feel that I can better process questions/commands/requests made to me, and can react appropriately with no delay or needing of repetition.

TABLE 3: Patient's Post-Lumbar Puncture - Normal Pressure Hydrocephalus 7 Day Log 


\section{Cureus}

\section{Day Day Day Day Day Day Day \\ \begin{tabular}{l|l|l|l|l|l|l|}
1 & 2 & 3 & 4 & 5 & 6 & 7
\end{tabular}}

1. The patient is more balanced.

2. The patient is more confident walking inside and outside.

3. The patient is better able to stand up and sit down.

4. The patient is better able to walk up and down stairs and hills.

5. The patient has more energy to complete their daily tasks.

6. The patient is better able to make plans, problem solve, and move from one task to the next.

7. The patient is better able to pay close and continuous attention to tasks.

8. The patient has more motivation to do daily chores and errands, call or see friends and family.

9. The patient enjoyed listening to music more.

10. The patient has fewer issues with their urinary urgency.

11. The patient can better process questions/commands/requests made to them, and react appropriately with no delay or needing of repetition

TABLE 4: Caregiver Post-Lumbar Puncture - Normal Pressure Hydrocephalus 7 Day $\log$

The principal outcome measures were the intention to treat with surgical shunt placement and shunt response. Intention to treat was expressed by the senior author after assessing the clinical and radiological presentation together with the results of the standard testing and the results of the new LP tool. Shunt response was defined using the same criteria as the European iNPH Multicentre Study $[9,11]$. Shunt response was recorded as the existence of improvement of at least five points in the European NPH scale between the presentation and the postoperative scores, respectively.

Categorical data was summarized using frequencies and percentages. Continuous data was summarized using median and interquartile range (IQR). Paired Wilcoxon's rank-sum test was used to assess differences before and after LP for continuous variables and Fisher's exact test was used for categorical variables. Logistic regression analysis was used to assess if there was a correlation between the studied scales and intention to treat as well as between the scales and shunt response. All reported $\mathrm{p}$-values were two-sided and statistical significance was set at $\mathrm{p}<$ 0.05. All analyses were performed using SAS version 9.3 (SAS Institute, Inc., Cary, NC, USA).

\section{Results}

\section{At presentation}




\section{Cureus}

Sixty-two patients were identified following application of the inclusion/exclusion criteria. Patient characteristics are shown in Table 5.

\section{Patient Characteristics at Presentation}

Age at Presentation, years (IQR)

Sex, Female, n (\%)

Race, n (\%)

Caucasian

African-American

Other

BMI, $\mathrm{kg} / \mathrm{m}^{2}$ (IQR)

Symptoms/Signs at Presentation, n (\%)

Gait/Balance Impairment

Urinary Urgency/Incontinence

Memory/Attention Deficits

Headaches/Dizziness

Presence of Complete Triad, n (\%)

Duration of Symptoms, months (IQR)

Physical Exam Scoring at Presentation

TUG, seconds (IQR)

Tinetti, median (IQR)

MCV Scale, median grade (IQR)

MMSE at presentation, median (IQR)

European Hydrocephalus Scale at presentation, points (IQR)

Evans Index at presentation, median (IQR)

Opening ICP, $\mathrm{cmH}_{2} \mathrm{O}$ (IQR)

\section{Patients $(\mathrm{N}=62)$}

$76(71-80)$

$26(42 \%)$

59 (95\%)

$2(3 \%)$

$1(2 \%)$

$27.6(23.9-31.5)$

$61(98 \%)$

$54(87 \%)$

48 (77\%)

$17(27 \%)$

$41(66 \%)$

$24(16-48)$

Pre-LP

Post-LP

$15(12-26)$

$14(12-20)$

$22(17-25)$

$24(20-26)$

$3(2-4)$

$2(2-3)$

27 (21-29)

$52(30-76)$

$0.36(0.34-0.37)$

$15(13-19)$

\section{TABLE 5: Patient Demographics}

Abbreviation: interquartile range: IQR; body mass index: BMI; Timed Up and Go: TUG; Medical College of Virginia gait grade: MCV; Mini-mental state examination: MMSE; intracranial pressure: ICP. 
Median age was 76 years. The complete Hakim's triad was present in $66 \%$ of cases. Examining the signs and symptoms separately, $98 \%$ of patients presented with gait or balance deterioration, $87 \%$ with urinary urgency or incontinence, and $77 \%$ with memory or attention deficits. The median Evans index was 0.36; median opening pressure was $15 \mathrm{~cm} \mathrm{H}_{2} \mathrm{O}$, and the median duration of symptoms was 24 months. At presentation, median TUG was 15 minutes; median Tinetti score was 22; median MCV grade was 3, and median European scale score was 52.

\section{Post LP changes}

TUG ( $\mathrm{p}<0.008)$, Tinetti $(\mathrm{p}<0.001)$, and MCV grade $(\mathrm{p}=0.01)$ significantly improved following LP. Overall, 57 (92\%) patients demonstrated post-LP log improvement. The post-LP log score improvement was statistically significant for both patients $(\mathrm{p}<0.001)$ and caregivers $(\mathrm{p}<0.001)$ independently and combined $(\mathrm{p}<0.001)$.

Interestingly, as shown in Figure 1, the responses to the LP log questionnaire showed a clear initial post-LP improvement with a linear slow return to the pre-LP status.

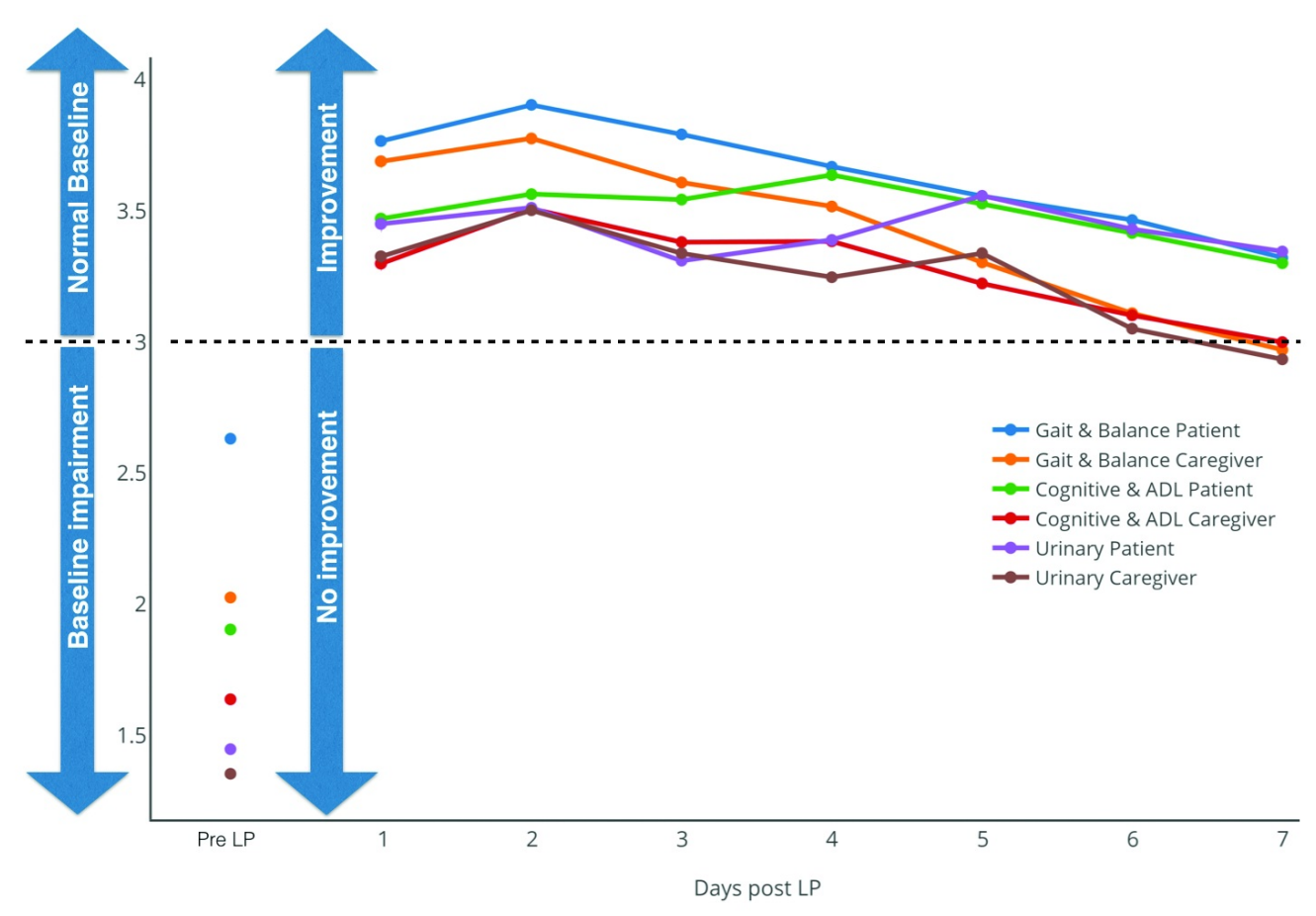

FIGURE 1: Baseline and post-LP log scores over time comparing the gait/balance, cognitive/ADL, and urinary domains of questions in patients and caregivers.

Abbreviations: Activities of daily living: ADL

Patients responded with significantly better performance than caregivers for all questions at both baseline and each day post-LP. The peak improvement, as assessed by the caregivers, was identified on the second-day post-LP for gait, urinary, and cognitive questions. However, the optimal temporal performance for patients in self-assessment was the second-day post-LP for gait, the fourth-day post-LP for cognition, and the fifth-day post-LP for urinary symptomatology. 


\section{Cureus}

Patients with significant post-LP log improvement had statistically significant improvement between pre- and post-LP TUG (mean difference: 2.88 seconds, $\mathrm{p}=0.01$ ) and Tinetti (mean difference: 2 points, $\mathrm{p}<0.0001$ ). Neither TUG nor Tinetti demonstrated any statistical differences between pre- and post-LP timing in patients that did not have post-LP log improvement.

Patients that had a good baseline gait scoring test (TUG $<=12$ seconds, Tinetti $>=25$, and MCV grade $<=3$ ) did not show significant statistical change of their post-LP TUG ( $p=0.41)$, post-LP Tinetti $(\mathrm{p}=1)$, or post-LP MCV $(\mathrm{p}=0.9)$. However, $90 \%$ of patients with good baseline gait scoring improved in their post-LP log. Figure 2 depicts the mean post-LP log scores in patients with good baseline gait performance.

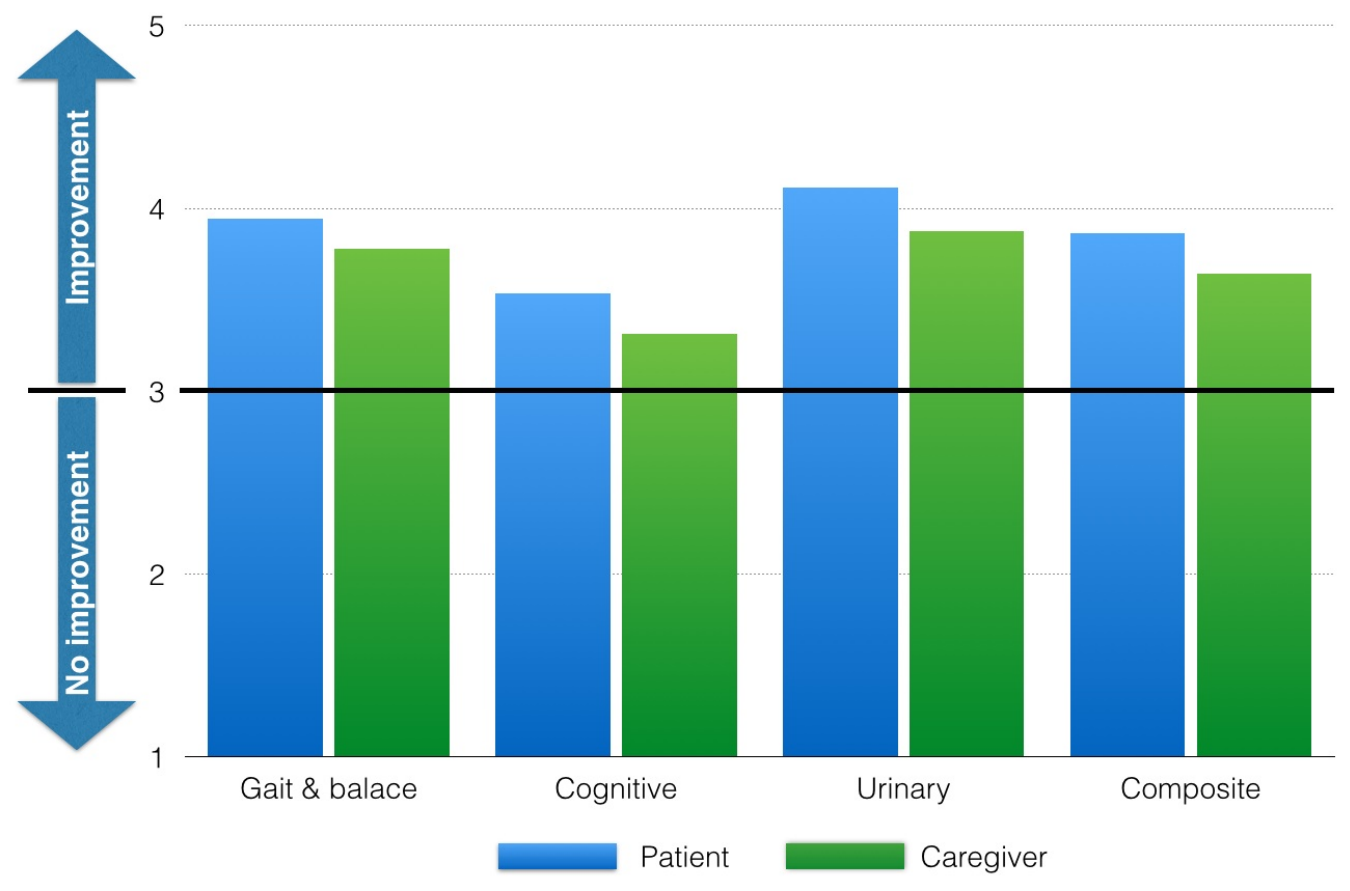

FIGURE 2: Post-LP log scores of patients with good baseline TUG, Tinetti, and MCV tests.

The LP log detected a post-LP improvement in all patients that did not have an evident change in their post-LP (TUG difference post-LP to pre-LP $<1$ second, Tinetti difference post-LP to pre-LP $<2$ points, and MCV grade difference $<1$ ). Figure 3 details the mean post-LP log scores in patients that did not have an evident change in their gait performance post-LP. 


\section{Cureus}

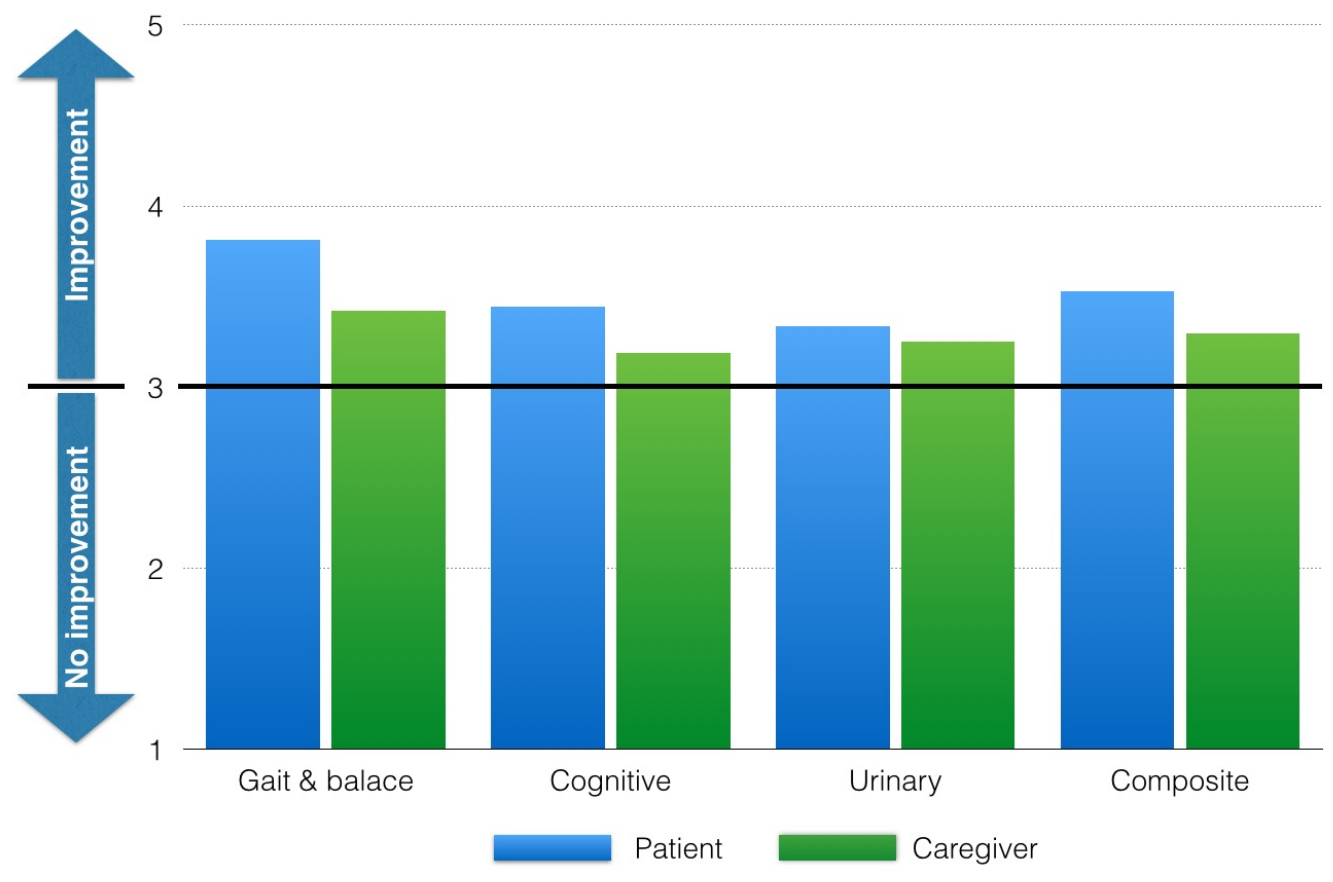

FIGURE 3: Post-LP log scores of patients without improvement in post-LP TUG, Tinetti, and MCV tests.

\section{Intention to treat}

Surgery was indicated in 53 (85\%) patients. Improvement of at least one second in pre-LP to post-LP TUG $(\mathrm{p}>0.05)$ and at least one point in pre-LP to post-LP Tinetti was not associated with a surgical indication ( $p>0.05$ ). Post-LP log improvement was significantly associated with surgical indication OR: 24.5 95\%CI (2.4 - 248.12), p = 0.0068), as shown in Table 6.

\begin{tabular}{|c|c|c|c|}
\hline Post-LP Improvement & OR & $95 \% \mathrm{Cl}$ & $p$-value \\
\hline TUG Improvement & 1.68 & $0.45-7.10$ & 0.451 \\
\hline Tinetti Improvement & 1.72 & $0.44-6.57$ & 0.42 \\
\hline MCV Improvement & 1.99 & $0.44-14.067$ & 0.41 \\
\hline LP Log Improvement & 24.50 & $2.4-248.12$ & 0.0068 \\
\hline
\end{tabular}

\section{TABLE 6: Univariate Logistic Regression Analysis for Intention to Treat}

Abbreviations: Timed Up and Go: TUG; Medical College of Virginia gait grade: MCV; Odds Ratio: OR; Confidence Interval: CI

Table 7 demonstrates the sensitivity, specificity, and diagnostic accuracy for intention to treat in the TUG, Tinetti, MCV, and LP Log responses. The assessment with the greatest sensitivity and diagnostic accuracy for indication to treat was the LP log. 


\section{Cureus}

\begin{tabular}{|c|c|c|c|}
\hline Intention to Treat & Sensitivity & Specificity & Accuracy \\
\hline TUG & $4 \%$ & $100 \%$ & $24 \%$ \\
\hline TInettI & $21 \%$ & $86 \%$ & $34 \%$ \\
\hline MCV & $66 \%$ & $29 \%$ & $58 \%$ \\
\hline LP Log & $98 \%$ & $33 \%$ & $85 \%$ \\
\hline Shunt Response & Sensitivity & Specificity & Accuracy \\
\hline TUG & $0 \%$ & $78 \%$ & $19 \%$ \\
\hline Tinetti & $19 \%$ & $89 \%$ & $36 \%$ \\
\hline MCV & $56 \%$ & $22 \%$ & $47 \%$ \\
\hline LP Log & $96 \%$ & $0 \%$ & $72 \%$ \\
\hline
\end{tabular}

TABLE 7: Sensitivity and Specificity of the Different Scales for Intention to Treat and Shunt Response

Abbreviations: Timed Up and Go: TUG; Medical College of Virginia gait grade: MCV

\section{Surgical outcomes}

Seventy-nine percent of shunted patients showed shunt response at their last follow-up (defined as at least five points of post-surgical improvement on the European NPH scale). Preand post-LP TUG, Tinetti, or MCV changes were not associated with shunt response ( $p>0.05)$. Association with post-surgical improvement could not be assessed due to a lack of patients that underwent surgery with a lack of improvement in the post-LP log, resulting in an LP log specificity of 0 (Table 3). However, the assessment with the greatest sensitivity and diagnostic accuracy for shunt response was the LP log.

\section{Discussion}

This study demonstrates how the newly described LP log can detect post-LP differences in patient function when traditional assessments, such as TUG, Tinetti, or MCV fail to do so. The LP log demonstrated better sensitivity, diagnostic accuracy, and association with surgical indication than the currently utilized diagnostic approaches, thus, validating use of the log to aid in the diagnosis of iNPH.

The objective diagnosis of iNPH is complicated by several factors. Idiopathic NPH takes several years to develop and the initial symptoms are soft and easy to miss [4, 12]. Patients with iNPH span a continuum from very healthy and functional to very sick and disabled. MRI scans have helped in the early recognition of ventriculomegaly, often preceding the appearance of any symptoms. At the other end of the spectrum, in the elderly population, comorbidities are much more pronounced and may mask iNPH symptomatology, especially if there is a slow progression [13-15]. 
It is crucial to have the ability to detect changes at earlier timepoint, as there are better outcomes with early treatment [6]. However, our currently used assessments lack sensitivity and often fail to detect significant changes at early phases. In our experience, an initial negative work-up often becomes positive following reassessment at a later time when symptoms have progressed. Deficits that were initially minor then became "measurable" with our tests, proving that our present diagnostic tools are lacking in the early phase and, therefore, treatment is delayed.

The situation may be even more dire in highly functional patients (athletic individuals or those with high IQ) with hydrocephalus. These patients face a greater hurdle, as even a significant decline in their "baseline" function often still falls in the "normal" range, because tests results are reported in comparison to a control population whose baseline level of function is often at a much lower level than the baseline function of these high-functioning individuals. This situation would therefore delay the detection of the early symptoms of iNPH for several years, often resulting in a drastic reduction in function before diagnosis.

Another issue is that current assessments are based on physical exams performed in a clinical environment. Family members and caregivers often report that patients are significantly improved clinic, compared to their daily life, which may be due to several factors. For instance, patients often try to demonstrate optimal performance to the healthcare team while being evaluated. Additionally, the clinic environment does not provide the same challenges as daily life. Therefore, to be relevant, iNPH diagnostic testing needs to be sensitive to the individual baseline functional status and needs to be sensitive enough to measure a variation from baseline. The new LP log compares the changes observed in the daily life of patients after CSF tapping for seven consecutive days, thus, providing longer-term evaluation of patient function following a tap test.

Interestingly, our results showed that the post-LP improvement peak was not the first day post-CSF tapping. Instead, the peak of improvement was most commonly reported by the caregiver on the second day, while the optimal performance for patients was the second day for gait, the fourth day for cognition, and the fifth day for urinary evaluation. This information may explain why differences may not be detected when measuring gait performance on the first day post-LP. This may also explain why it is possible to detect differences in other patients following several days of external lumbar drainage, although this requires hospital admission for several days with increased costs and an increased risk of patient morbidity. This information again highlights the critical importance of longer-term evaluation of patient function following a tap test, which would be challenging in an entirely clinic-based assessment.

\section{Conclusions}

The LP log showed better sensitivity, diagnostic accuracy, and association with surgical indication than the current diagnostic approach. The LP log may be useful detecting NPH patients at earlier stages and, therefore, yielding better surgical outcomes. Additionally, as it is a patient and caregiver-based assessment, there is limited additional healthcare expenditure.

\section{Additional Information}

\section{Disclosures}

Human subjects: Consent was obtained by all participants in this study. Institutional Review Board issued approval NA_00044584. Animal subjects: All authors have confirmed that this study did not involve animal subjects or tissue. Conflicts of interest: In compliance with the ICMJE uniform disclosure form, all authors declare the following: Payment/services info: All 
authors have declared that no financial support was received from any organization for the submitted work. Financial relationships: All authors have declared that they have no financial relationships at present or within the previous three years with any organizations that might have an interest in the submitted work. Other relationships: All authors have declared that there are no other relationships or activities that could appear to have influenced the submitted work.

\section{Acknowledgements}

Ignacio Jusué-Torres, M.D. receives research support from the Salisbury Hydrocephalus Research Fellowship grant and the Swenson Family Foundation. Daniele Rigamonti, M.D. receives research support from the Salisbury Family Foundation The abstract for this manuscript was presented as a poster at the Annual Meeting of the Congress of Neurological Surgeons in New Orleans, Louisiana on September 26-30, 2015. This poster was then honored as the Top Poster in the Stereotactic and Functional section.

\section{References}

1. Hakim S, Adams RD: The special clinical problem of symptomatic hydrocephalus with normal cerebrospinal fluid pressure. Observations on cerebrospinal fluid hydrodynamics. J Neurol Sci. 1965, 2:307-27. 10.1016/0022-510X(65)90016-X

2. Adams RD, Fisher CM, Hakim S, Ojemann RG, Sweet WH: Symptomatic occult hydrocephalus with "normal" cerebrospinal-fluid pressure - A treatable syndrome. N Engl J Med. 1965, 273:117-26. 10.1056/NEJM196507152730301

3. Meier U, Zeilinger FS, Kintzel D: Signs, symptoms and course of normal pressure hydrocephalus in comparison with cerebral atrophy. Acta Neurochir (Wien). 1999, 141:103948. 10.1007/s007010050480

4. McGirt MJ, Woodworth G, Coon AL, Thomas G, Williams MA, Rigamonti D: Diagnosis, treatment, and analysis of long-term outcomes in idiopathic normal-pressure hydrocephalus. Neurosurgery. 2005, 57:699-705. 10.1227/01.NEU.0000175724.00147.10

5. Hellström P, Klinge P, Tans J, Wikkelsø C: A new scale for assessment of severity and outcome in iNPH. Acta Neurol Scand. 2012, 126:229-37. 10.1111/j.1600-0404.2012.01677.x

6. Andren K, Wikkelso C, Tisell M, Hellstrom P: Natural course of idiopathic normal pressure hydrocephalus. J Neurol Neurosurg Psychiatry. 2014, 85:806-10. 10.1136/jnnp-2013-306117

7. Iseki C, Kawanami T, Nagasawa H, Wada M, Koyama S, Kikuchi K, Arawaka S, Kurita K, Daimon M, Mori E, Kato T: Asymptomatic ventriculomegaly with features of idiopathic normal pressure hydrocephalus on MRI (AVIM) in the elderly: a prospective study in a Japanese population. J Neurol Sci. 2009, 277:54-57. 10.1016/j.jns.2008.10.004

8. Tisell M, Tullberg M, Hellstrom P, Edsbagge M, Hogfeldt M, Wikkelso C: Shunt surgery in patients with hydrocephalus and white matter changes. J Neurosurg. 2011, 114:1432-38. 10.3171/2010.11.JNS10967

9. Wikkelso C, Hellstrom P, Klinge PM, Tans JT: The European iNPH Multicentre Study on the predictive values of resistance to CSF outflow and the CSF Tap Test in patients with idiopathic normal pressure hydrocephalus. J Neurol Neurosurg Psychiatry. 2013, 84:562-68. 10.1136/jnnp-2012-303314

10. Sankey EW, Jusue-Torres I, Elder BD, Goodwin CR, Batra S, Hoffberger J, Lu J, Blitz AM, Rigamonti D: Functional gait outcomes for idiopathic normal pressure hydrocephalus after primary endoscopic third ventriculostomy. J Clin Neurosci. 2015, 22:1303-1308.

10.1016/j.jocn.2015.02.019

11. Klinge P, Hellström P, Tans J, Wikkelsø C; European iNPH Multicentre Study Group: One-year outcome in the European multicentre study on iNPH. Acta Neurol Scand. 2012, 126:145-53. 10.1111/j.1600-0404.2012.01676.x

12. Mori E, Ishikawa M, Kato T, Kazui H, Miyake H, Miyajima M, Nakajima M, Hashimoto M, Kuriyama N, Tokuda T, Ishii K, Kaijima M, Hirata Y, Saito M, Arai H: Guidelines for management of idiopathic normal pressure hydrocephalus: second edition. Neurol Med Chir (Tokyo). 2012, 52:775-809. 10.2176/nmc.52.775

13. Gallia GL, Rigamonti D, Williams MA: The diagnosis and treatment of idiopathic normal 


\section{Cureus}

pressure hydrocephalus. Nat Clin Pract Neurol. 2006, 2:375-81. 10.1038/ncpneuro0237

14. Shprecher D, Schwalb J, Kurlan R: Normal pressure hydrocephalus: diagnosis and treatment . Curr Neurol Neurosci Rep. 2008, 8:371-76. 10.1007/s11910-008-0058-2

15. Malm J, Graff-Radford NR, Ishikawa M, Kristensen B, Leinonen V, Mori E, Owler BK, Tullberg M, Williams MA, Relkin NR: Influence of comorbidities in idiopathic normal pressure hydrocephalus - research and clinical care. A report of the ISHCSF task force on comorbidities in INPH. Fluids Barriers CNS. 2013, 10:22. 10.1186/2045-8118-10-22 